\title{
Outstanding Reviewers for RSC Advances in 2019
}

Cite this: RSC Adv., 2020, 10, 18581

DOI: 10.1039/d0ra90038a

rsc.li/rsc-advances

We would like to take this opportunity to thank all of RSC Advances' reviewers, and in particular highlight the Outstanding Reviewers for the journal in 2019, as selected by the editorial team, for their significant contribution to RSC Advances. We would also like to direct a special thanks to the members of the $R S C$ Advances Reviewer Panel for their hard work and dedication, and the valuable contribution they have made to the journal. The Reviewer Panel is a key part of our commitment to deliver rigorous and fair peer review and ensures that manuscripts are handled by experts throughout the peer review process. We are proud to work with these individuals and recognise their crucial role for the journal.

We announce our Outstanding Reviewers annually and each receives a certificate to give recognition for their contribution. The reviewers have been chosen based on the number, timeliness and quality of the reports completed over the year.

\section{RSC Advances 2019 Outstanding Reviewers:}

Dr Arghya Adhikary

University of Calcutta

Professor Katsuhiko Ariga

Busshitsu Zairyo Kenkyu Kiko

ORCID: 0000-0002-2445-2955
Dr Yaocai Bai

University of California Riverside

ORCID: 0000-0003-3700-2520

Dr Xi Chen

Shanghai Jiao Tong University

Dr Anindita Das

Indian Association for the Cultivation

of Science (IACS)

ORCID: 0000-0001-8723-6291

Dr Nilesh Gaikwad

Gaikwad Steroidomics Lab LLC

ORCID: 0000-0002-4990-4508

Professor Wei-Min $\mathrm{He}$

Hunan University of Science and Engineering

ORCID: 0000-0002-9481-6697

Dr Mark Honey

University of Greenwich

ORCID: 0000-0001-7272-476X

Dr Dattatray Late

National Chemical Laboratory CSIR

ORCID: 0000-0003-3007-7220

Dr Giuseppe Lazzara

University of Palermo

ORCID: 0000-0003-1953-5817

Dr Samir Nuseibeh

University College London

ORCID: 0000-0003-1787-636X
Dr Veli Ozalp

Konya Gida ve Tarim Universitesi

Dr Qingsen Shang

University of Michigan

ORCID: 0000-0002-6782-3437

Dr Diptesh Sil

Atul Ltd

ORCID: 0000-0001-6457-0545

Dr Steven Suib

University of Connecticut

Dr Balaram Takale

University of California Santa Barbara

Dr Xiaobin Wu

Shanghai Normal University

Dr Murat Yavuz

Dicle Universitesi

ORCID: 0000-0003-3452-8551

Professor Wenwu Zhong

Taizhou University

RSC Advances Reviewer Panel 2019 Outstanding Reviewers:

Dr Vipul Agarwal

University of New South Wales

ORCID: 0000-0002-6239-5410

Dr Ashootosh Ambade

National Chemical Laboratory CSIR

ORCID: 0000-0003-3605-5719 
Dr Rok Borstnar

Kemijski institute

ORCID: 0000-0002-6786-5434

Professor Lingxin Chen

Chinese Academy of Sciences

ORCID: 0000-0002-3764-3515

Dr Ummadisetti Chinnarajesh Indiana University Bloomington

ORCID: 0000-0002-0065-2223

Dr Emanuele Curotto

Arcadia University

Dr Serap Evran

Ege Universitesi

ORCID: 0000-0001-6676-4888

Dr Nicholas Geitner

Duke University

ORCID: 0000-0003-4313-372X

Dr Prokopios Georgopanos Helmholtz-Zentrum Geesthacht Zentrum fur Materialforschung und Kustenforschung

ORCID: 0000-0002-6394-0628
Dr S. Girish Kumar

CMR University

ORCID: 0000-0001-9132-1202

Dr Hu Li

Guizhou University

ORCID: 0000-0003-3604-9271

Dr Jianmin Li

Zhejiang University

ORCID: 0000-0002-3917-8653

Dr Shiwei Qu

Scripps Research Institute

ORCID: 0000-0002-9358-066X

Dr Leo Small

Sandia National Laboratories

ORCID: 0000-0003-0404-6287

Professor David Thompson

Sam Houston State University

ORCID: 0000-0002-2934-5729

Dr Maria Timofeeva

FGBUN Institut kataliza im G. K. Boreskova Sibirskogo otdelenia Rossijskoj akademii nauk
Dr Paul Trippier

University of Nebraska Medical Center

ORCID: 0000-0002-4947-5782

Dr Mark Waterland

Massey University

ORCID: 0000-0002-8493-9407

Professor Chunping Yang

Hunan University

ORCID: 0000-0003-3987-2722

Professor Lei Yu

Yangzhou University

We would also like to thank the $R S C$ Advances Editorial Board, Advisory Board and the research community for their continued support of the journal, as authors, reviewers and readers.

Laura Fisher, Executive Editor

Russell Cox, Editor-in-Chief 